\title{
3D Dynamic Programming Approach to Functional Equations with Applications
}

\author{
Awais Asif, ${ }^{1}$ Sami Ullah Khan $\mathbb{D}^{2}{ }^{2}$ Thabet Abdeljawad $\mathbb{D}^{3,4,5}$ Muahammad Arshad, ${ }^{1}$ \\ and Amjad Ali ${ }^{1}$ \\ ${ }^{1}$ Department of Math \& Stats, International Islamic University Islamabad, Pakistan \\ ${ }^{2}$ Department of Mathematics, Gomal University, D.I.Khan, 29050, KPK, Pakistan \\ ${ }^{3}$ Department of Mathematics and General Sciences, Prince Sultan University, P.O. Box 66833, Riyadh, Saudi Arabia \\ ${ }^{4}$ Department of Medical Research, China Medical University, Taichung 40402, Taiwan \\ ${ }^{5}$ Department of Computer Science and Information Engineering, Asia University, Taichung 40402, Taiwan
}

Correspondence should be addressed to Sami Ullah Khan; gomal85@gmail.com and Thabet Abdeljawad; tabdeljawad@psu.edu.sa Received 17 July 2020; Revised 10 August 2020; Accepted 28 August 2020; Published 21 September 2020

Academic Editor: Zoran Mitrovic

Copyright (c) 2020 Awais Asif et al. This is an open access article distributed under the Creative Commons Attribution License, which permits unrestricted use, distribution, and reproduction in any medium, provided the original work is properly cited.

This article demonstrates the graphical existence of a single fixed point while imposing the contractive condition of Chatterjea type $F$-contraction on $F$-metric space (briefly as $F$-MS). We present two examples that verify the validity of the results given in the paper. The paper further explains the subsistence of the fixed point even if the contractive condition is valid only for a closed ball inside the space rather than imposing it on the whole F-MS. Moreover, the application of the mentioned results in finding a single solution of functional equations is described that is widely used in computer programming and optimization.

\section{Introduction and Preliminaries}

After Banach presented his renowned Banach Contraction Principle, his idea was generalized by various authors into many interesting generalizations (see [1-8]). Wardowski [9] extended his idea to a more generalized form which he named as F-contraction. An additional strictly increasing function $F$ with certain other restrictions was used to modify the Banach theorem. He investigated the fixed point of the contraction and explained the generality of his theorem with the help of concrete examples. This idea was furthered by Klim and Wardowski [10] into set-valued maps using a dynamic process instead of the ordinary Picard sequence. Later, Nazam et al. [11] extended Wardowski's theorem into the form of Kannan's theorem and hence proved the theorem for noncontinuous maps. He also described that a fixed point for such maps can be iterated even if the contractive inequality holds true only for a subset closed ball of the MS. The notion of $F$-contraction was extended by other authors as well (see [12-16]).
This article relaxes the map $F$ by eliminating one of its restrictions, (F3) and hence iterates a fixed point for it. The investigation is carried out for single as well as set-valued maps. Our work is new which extends the preexisting theorems of Wardowski and their consequent results. This paper demonstrates the main idea of this research with the help of graphs which unifies work from the previous research carried out on the topic.

Some basic definitions are given below which will be needed in a sequel.

Definition 1 (see [17]). Assume $\mathscr{G}$ is a set of functions $g:(0$, $+\infty) \longrightarrow R$ satisfying the following conditions:

(F1) $g$ is a nondecreasing function, i.e., $0<u<v \Longrightarrow$ $g(u) \leq g(v)$,

(F2) for any sequence $t_{n} \subset(0,+\infty)$, we have

$$
\lim _{n \rightarrow \infty} t_{n}=0 \Leftrightarrow \lim _{n \rightarrow \infty} g\left(t_{n}\right)=-\infty
$$


Definition 2 (see [17]). Assume a nonempty set $E$ and $d: E$ $\times E \longrightarrow[0, \infty)$ is a map. Assume that there is some $(g, \sigma)$ $\in \mathscr{G} \times[0,+\infty)$ such that

(d1) $(a, x) \in E \times E, d(a, x)=0 \Leftrightarrow a=x,(a, x) \in E \times E, d$ $(a, x)=d(x, a)$,

(d2) $(a, x) \in E \times E, d(a, x)=d(x, a)$

(d3) for every $(a, x) \in E \times E$, for each $N^{\prime} \in N, N^{\prime} \geq 2$, and for every $\left(t_{i}\right)_{i=1}^{n} \subset E$ with $\left(t_{1}, t_{N^{\prime}}\right)=(a, x)$, we have $(a, x)>0$ $\Longrightarrow g(d(a, x)) \leq g\left(\sum_{i=1}^{N^{\prime}-1} d\left(t_{i}, t_{i+1}\right)\right)+\sigma$.

Then $d$ is called an $F$-metric on $A$, while $(E, d)$ is named as $F$-MS.

Example 3 (see [17]). Let $E=N$ and $d: E \times E \longrightarrow(0, \infty)$ be defined by

$$
d(a, x)= \begin{cases}(a-x)^{2}, & \text { if }(a, x) \in[0,3] \times[0,3], \\ |a-x|, & \text { if }(a, x) \notin[0,3] \times[0,3],\end{cases}
$$

for all $(a, x) \in E \times E$. Then, $d$ is an F-MS.

Example 4 (see [17]). Let $E=N$ and $d: E \times E \longrightarrow(0, \infty)$ is defined as

$$
d(a, x)= \begin{cases}0, & \text { if } a=x, \\ e^{|a-x|}, & \text { if } a \neq x,\end{cases}
$$

for all $(a, x) \in E \times E$. Then, $d$ is an $F$-metric on $E$.

Definition 5 (see [17]). Let $\left(a_{n}\right) \in$ E. If

(i) $\lim _{n \rightarrow \infty} d\left(a_{n}, a\right)=0$ for some $a \in E$. Then $\left(a_{n}\right)$ is $F$-convergent to $a$

(ii) $\lim _{n, m \rightarrow \infty} d\left(a_{n}, a_{m}\right)=0$ then the sequence $\left(a_{n}\right)$ is F-Cauchy

(iii) For each $\left(a_{n}\right) \subset E$ implies $\left(a_{n}\right)$ is $F$-convergent. Then, the space $(E, d)$ is known as $F$-complete

Definition 6 (see [17]). Let $(E, d)$ be an F-MS. A subset $O$ of $E$ is said to be $F$-open if, for every $a \in O$, there is some $r>0$ such that $B(a, r) \subset O$, where

$$
B(a, r)=\{x \in E: d(a, x)<r\} .
$$

We say that a subset $C$ of $E$ is $F$-closed if $E \backslash C$ is $F$-open.

Definition 7 (see [17]). Let $B$ be a nonempty subset of $E$ and $d$ be an $F$-metric, then, the following statements are equivalent:

(i) $B$ is $F$-closed

(ii) For any sequence $\left(a_{n}\right) \subset B$, we have

$$
\lim _{n \rightarrow \infty} d\left(a_{n}, a\right)=0, a \in E \Longrightarrow a \in B
$$

Theorem 8 (see [17]). Assume (E,d) is an F-complete F-MS, and let $h: E \longrightarrow E$ be a given map. Let there is some $k \in(0,1)$ such that

$$
d(h(a), h(x)) \leq k d(a, x),(a, x) \in E \times E .
$$

Then, $h a^{*}=a^{*}$ for at most one $a^{*} \in E$. Moreover, for any $a_{0} \in E$, the sequence $\left(a_{n}\right) \subset E$ defined by $a_{n+1}=h\left(a_{n}\right), n \in N$ is F-convergent to $a^{*}$.

Theorem 9 (see [8]). Assume that E is a complete MS with metric $d$, and consider $h: E \longrightarrow E$ be a function such that

$$
d(h(a), h(x)) \leq \alpha d(a, x)+\beta d(a, h(x))+\gamma d(a, h(x)),
$$

for all $a, x \in E$, where $\alpha, \beta$, and $\gamma$ are nonnegative numbers satisfying $\alpha+\beta+\gamma<1$. Then, $h$ has a unique fixed point.

Lemma 10 (see [18]). Let $(B(W),\|\cdot\|)$ is a Banach space and $d$ is a metric defined by

$$
d(J, h)=\|J-h\|=\max _{a \in W}|J(a)-h(a)|, \quad J, h \in B(W) .
$$

Then, $(B(W),\|\cdot\|)$ is an F-MS.

\section{Common Fixed Points Results of Reich Type F-Contractions}

This section of the paper investigates the fixed point of single-valued $F$-contractions for two maps and single map in F-MS.

Theorem 11. Assume $F \in \mathscr{G},(X, d)$ is an F-complete F-MS and $S, T: X \longrightarrow X$ are self-mappings. Assume that for nonnegative functions $\alpha$ and $\beta$ with $\max _{a, x \in X}\{\alpha(a, x)+2 \beta(a, x)\}<1$, there is some $\tau>\sigma>0$ such that

$\tau+F(d(S a, T x)) \leq F[\alpha(a, x) d(a, x)+\beta(a, x)\{d(x, S a)+d(a, T x)\}]$,

with $\min \{d(S a, T x), d(a, x)\}>0$, for all $(a, x) \in X \times X$. Then, $S y=T y=y$ for some $y$ in $X$.

Proof. Choose an arbitrary point $a_{0}$ and iterate a sequence $\left(a_{n}\right)$ by

$$
S a_{2 c}=a_{2 c+1} \text { and } T a_{2 c+1}=a_{2 c+2} ; c=0,1,2, \cdots
$$


Using (9) and (10), we can write

$$
\begin{aligned}
\tau+F( & \left.d\left(a_{2 c+1}, a_{2 c+2}\right)\right) \\
= & +F\left(d\left(S a_{2 c}, T a_{2 c+1}\right)\right) \\
\leq & F\left[\alpha\left(a_{2 c}, a_{2 c+1}\right) d\left(a_{2 c}, a_{2 c+1}\right)+\beta\left(a_{2 c}, a_{2 c+1}\right)\right. \\
& \left.\cdot\left\{d\left(a_{2 c+1}, S a_{2 c}\right)+d\left(a_{2 c}, T a_{2 c+1}\right)\right\}\right] \\
= & F\left[\alpha\left(a_{2 c}, a_{2 c+1}\right) d\left(a_{2 c}, a_{2 c+1}\right)+\beta\left(a_{2 c}, a_{2 c+1}\right)\left(a_{2 c}, a_{2 c+2}\right)\right] \\
\leq & F\left[\alpha\left(a_{2 c}, a_{2 c+1}\right) d\left(a_{2 c}, a_{2 c+1}\right)+\beta\left(a_{2 c}, a_{2 c+1}\right)\right. \\
& \left.\cdot\left\{\left(a_{2 c}, a_{2 c+1}\right)+\left(a_{2 c+1}, a_{2 c+2}\right)\right\}\right]+\sigma .
\end{aligned}
$$

Using (F1), we have

$$
\begin{aligned}
d\left(a_{2 c+1}, a_{2 c+2}\right)< & \frac{\alpha\left(a_{2 c}, a_{2 c+1}\right)+\beta\left(a_{2 c}, a_{2 c+1}\right)}{1-\beta\left(a_{2 c}, a_{2 c+1}\right)} d\left(a_{2 c}, a_{2 c+1}\right) \\
= & \lambda_{1} d\left(a_{2 c}, a_{2 c+1}\right), \\
& \text { say } \frac{\alpha\left(a_{2 c}, a_{2 c+1}\right)+\beta\left(a_{2 c}, a_{2 c+1}\right)}{1-\beta\left(a_{2 c}, a_{2 c+1}\right)}=\lambda_{1} .
\end{aligned}
$$

Similarly,

$$
\begin{aligned}
d\left(a_{2 c+2}, a_{2 c+3}\right) & <\frac{\alpha\left(a_{2 c+1}, a_{2 c+2}\right)+\beta\left(a_{2 c+1}, a_{2 c+2}\right)}{1-\beta\left(a_{2 c+1}, a_{2 c+2}\right)} d\left(a_{2 c+1}, a_{2 c+2}\right) \\
& =\lambda_{2} d\left(a_{2 c+1}, a_{2 c+2}\right) .
\end{aligned}
$$

Hence, for $\lambda=\max \left\{\lambda_{1}, \lambda_{2}, \cdots, \lambda_{l}\right\}$, we have

$$
d\left(a_{n}, a_{n+1}\right)<\lambda d\left(a_{n-1}, a_{n}\right) \quad \text { for all } n \in N \text {, }
$$

which yields

$$
\begin{aligned}
d\left(a_{n}, a_{n+1}\right) & <\lambda d\left(a_{n-1}, a_{n}\right)<\lambda^{2} d\left(a_{n-2}, a_{n-1}\right)<\cdots \\
& <\lambda^{n} d\left(a_{0}, a_{1}\right), n \in N
\end{aligned}
$$

Using (15), we can write

$$
\begin{aligned}
\sum_{k=n}^{m-1} d\left(a_{k}, a_{k+1}\right) & <\lambda^{n}\left[1+\lambda+\lambda^{2}+\cdots+\lambda^{m-n-1}\right] d\left(a_{0}, a_{1}\right) \\
& \leq \frac{\lambda^{n}}{1-\lambda} d\left(a_{0}, a_{1}\right), m>n .
\end{aligned}
$$

Since $\lim _{n \rightarrow \infty}\left(\lambda^{n} / 1-\lambda\right) d\left(a_{0}, a_{1}\right)=0$, for any $\delta>0, \exists$ some $n^{\prime} \in N$ such that

$$
0<\frac{\lambda^{n}}{1-\lambda} d\left(a_{0}, a_{1}\right)<\delta, n \geq n^{\prime}
$$

Further, let $(g, \sigma) \in \mathscr{G} \times[0, \infty)$ satisfies (d3) and $\epsilon>0$ is fixed. By (F2), there is some $\delta>0$ such that

$$
0<t<\delta \Longrightarrow g(t)<g(\epsilon)-\sigma
$$

By (17) and (18), we write

$$
g\left(\sum_{k=n}^{m-1} d\left(a_{k}, a_{k+1}\right)\right) \leq g\left(\frac{\lambda^{n}}{1-\lambda} d\left(a_{0}, a_{1}\right)\right)<g(\epsilon)-\sigma, m>n \geq n^{\prime} .
$$

Using the above equation and (d3), we have

$$
d\left(a_{n}, a_{m}\right)>0, m>n>n^{\prime} \Longrightarrow g\left(d\left(a_{n}, a_{m}\right)\right)<g(\epsilon) .
$$

This shows

$$
d\left(a_{n}, a_{m}\right)<\epsilon, m>n \geq n^{\prime}
$$

Hence, $\left(a_{n}\right)$ is F-Cauchy in $X$. Since $(X, d)$ is $F$-complete, $\exists y \in X$ such that $\left(a_{n}\right)$ is $F$-convergent to $y$, i.e.,

$$
\lim _{n \rightarrow \infty} d\left(a_{n}, y\right)=0
$$

Now, assume that $d(S y, y)>0$. Then,

$$
\tau+F\left(d\left(S y, a_{2 c+2}\right)\right) \leq F\left[\begin{array}{c}
\alpha\left(y, a_{2 c+1}\right) d\left(y, a_{2 c+1}\right) \\
+\beta\left(y, a_{2 c+1}\right)\left\{d\left(a_{2 c+1}, S y\right)+d\left(y, a_{2 c+2}\right)\right\}
\end{array}\right] .
$$

By (F1) and letting $c \longrightarrow \infty$, we have

$$
\left(1-\beta\left(y, a_{2 c+1}\right)\right) d(S y, y)<0,
$$

which is a contradiction, as $\beta(a, x)$ is nonnegative. Hence, $d(S y, y)=0$, i.e., $S y=y$.

Following the same steps, we get $T y=y$. Hence, $T y=$ Sy $=y$.

Uniqueness: assume another common fixed $z$ of the maps $S$ and $T$ and $y \neq z$.Then

$$
\begin{aligned}
\tau+F(d(y, z)) & =F(d(S y, T z)) \\
& \leq F\left[\begin{array}{c}
\alpha(y, z) d(y, z) \\
+\beta(y, z)\{d(z, S y)+d(y, T z)\}
\end{array}\right] \\
& =F\left[\begin{array}{c}
\alpha(y, z) d(y, z) \\
+\beta(y, z)\{d(z, y)+d(y, z)\}
\end{array}\right] .
\end{aligned}
$$

Using (F1), we get $(1-\alpha(y, z)-2 \beta(y, z)) d(y, z)<0$, which is a contradiction. Hence, $y=z$.

Example 12. Assume that $X=X_{c}:=\{6 c+2 / 3, c \in N\}$,

$$
d\left(X_{c}, X_{k}\right)=\left\{\begin{array}{ll}
0, & \text { if } X_{c}=X_{k}, \\
e^{\left|X_{c}-X_{k}\right|}, & \text { if } X_{c} \neq X_{k},
\end{array} \quad F\left(X_{c}\right)=\ln \left(X_{c}\right),\right.
$$


and $S, T: X \longrightarrow X$ are defined by

$$
T X_{c}= \begin{cases}X_{1}, & \text { if } c=1,2 \\ X_{c-1}, & \text { if } c>2\end{cases}
$$

and

$$
S X_{n}= \begin{cases}X_{1}, & \text { if } c=1, \\ X_{2}, & \text { if } c=2, \\ X_{c-2}, & \text { if } c>4 .\end{cases}
$$

On the other hand, we define $\alpha, \beta: X \times X \longrightarrow[0, \infty)$ by

$$
\begin{aligned}
& \alpha\left(X_{c}, X_{k}\right)= \begin{cases}0 & \text { if } X_{c}=X_{k}, \\
e^{-1 / 2} & \text { if } X_{c} \neq X_{k},\end{cases} \\
& \beta\left(X_{c}, X_{k}\right)=\left\{\begin{array}{ll}
e^{-2}, & \text { if } X_{k}=S X_{c} \\
0, & \text { otherwise. }
\end{array} \text { and } X_{c}=T X_{k},\right.
\end{aligned}
$$

One can verify that $F$ fulfill conditions (F1) and (F2) and that $d$ is an $F$-metric. Assume $c \neq k$, then

$$
\begin{aligned}
F\left(d\left(S X_{c}, T X_{k}\right)\right) & =\ln \left(e^{\left|X_{c-2}-X_{k-1}\right|}\right)=\ln \left(e^{|2(c-k)-2|}\right) \\
& <\ln \left(e^{-1 / 2} \cdot e^{|2(c-k)|}\right)=F\left(\alpha\left(X_{c}, X_{k}\right) d\left(X_{c}, X_{k}\right)\right) \\
& <F\left[\begin{array}{c}
\alpha\left(X_{c}, X_{k}\right) d\left(X_{c}, X_{k}\right) \\
+\beta\left(X_{c}, X_{k}\right)\left\{d\left(X_{k}, S X_{c}\right)+d\left(X_{c}, T X_{k}\right)\right\}
\end{array}\right],
\end{aligned}
$$

whenever $\min \left\{d\left(S X_{c}, T X_{k}\right), d\left(X_{c}, X_{k}\right)\right\}>0$. One can verify that $\max _{X_{c}, X_{k}}\left\{\alpha\left(X_{c}, X_{k}\right)+2 \beta\left(X_{c}, X_{k}\right)\right\}<1$. For $\tau \in\left(\sigma, \ln \left(e^{-1 / 2}\right.\right.$. $\left.\left.e^{|2(c-k)|} / e^{|2(c-k)-2|}\right)\right)=(\sigma, \ln (\sqrt[3]{e}))$, the inequality (9) is true. Furthermore, $X_{1}$ is a unique point such that $S X_{1}=T X_{1}=$ $X_{1}$.

Choosing $\alpha(a, x)=0$ in the previous result, a result of Chatterjea type $F$-contraction is obtained.

Corollary 13. Assume $F \in \mathscr{G},(X, d)$ is an F-complete F-MS and $S, T: X \longrightarrow X$ are self-maps. Assume that for $\beta: X \times X$ $\longrightarrow[0,1)$, there is some $\tau>\sigma$ such that

$$
\tau+F(d(S a, T x)) \leq F\left[\frac{\beta(a, x)}{2}(d(x, S a)+d(a, T x))\right]
$$

with $\min \{d(S a, T x), d(a, S a), d(x, T x)\}>0$, for all $(a, x) \in$ $X \times X$. Then, $T y=S y=y$ for a unique $y$ in $X$.

Substituting $S$ with $T$ in Corollary 13, we obtain the following result.
Corollary 14. Assume that $(X, d)$ is an F-complete $F$ $M S, F \in \mathscr{G}$ and $T: X \longrightarrow X$ is a self-map. Assume that for $\beta$ $: X \times X \longrightarrow[0,1)$, there is some $\tau>\sigma$ such that

$$
\tau+F(d(T a, T x)) \leq F\left[\frac{\beta(a, x)}{2}(d(a, T x)+d(x, T a))\right]
$$

with $\min \{d(T a, T x), d(a, T a)+d(x, T x)\}>0$, for all $(a, x)$ $\in X \times X$. Then, $T$ has at most one fixed point in $X$.

\section{Investigation of Fixed Points of $F$ -Contractions on F-Closed Balls}

This section of the paper investigates a fixed point of singlevalued $F$-contractions for two maps and single map imposed only on a $F$-closed subset of F-MS.

Definition 15. Assume an F-MS $(X, d)$ which is F-complete, $F \in \mathscr{G}$, and $S, T: X \longrightarrow X$ are self-maps, let $\alpha, \beta$ be nonnegative functions with $\max _{a, x \in X}\{\alpha(a, x)+2 \beta(a, x)\}<1$. Then, $T$ is named as Reich type $F$-contraction on $B\left(a_{0}, r\right) \subseteq X$ if there is some $\tau>\sigma$ satisfying

$$
\begin{aligned}
\tau & +F(d(S a, T x)) \\
& \leq F\left[\begin{array}{c}
\alpha(a, x) d(a, x) \\
+\beta(a, x)\{d(x, S a)+d(a, T x)\}
\end{array}\right], \forall a, x \in B\left(a_{0}, r\right) .
\end{aligned}
$$

Theorem 16. Assume $(g, \sigma) \in \mathscr{G} \times[0, \infty)$, an F-MS $(X, d)$ which is F-complete and $T$ is a Reich type F-contraction on $B\left(a_{0}, r\right)$. Assume that for $a_{0} \in X$ and $r>0$, the conditions given below are fulfilled:

(a) $B\left(a_{0}, r\right)$ is F-closed

(b) $d\left(a_{0}, a_{1}\right) \leq(1-\lambda) r$, for $a_{1} \in X$ and $\lambda=\alpha(a, x)+\beta$ $(a, x) / 1-\beta(a, x)$

(c) $\exists 0<\epsilon<r$ such as $g\left(\left(1-\lambda^{k+1}\right) r\right) \leq g(\epsilon)-\sigma$, where $k \in N$

Then, $T y^{*}=S y=y$ for some $y$ in $B\left(a_{0}, r\right)$.

Proof. Choose and arbitrary point $a_{0}$ and iterate a sequence $\left(a_{n}\right)$ by

$$
T a_{2 c}=a_{2 c+1} \text { and } \mathrm{S} a_{2 c+1}=a_{2 c+2} ; c=0,1,2, \cdots
$$

Using mathematical induction, we show that $a_{n}$ is in $B\left(a_{0}, r\right)$ for all $n \in N$. By hypothesis

$$
d\left(a_{0}, a_{1}\right)<r
$$


Therefore, $a_{1} \in B\left(a_{0}, r\right)$. Assume $a_{2}, \cdots, a_{k} \in B\left(a_{0}, r\right)$ for some $k \in N$. Now, if $a_{2 c+1} \leq a_{k}$, then by (33), we can write

$$
\begin{aligned}
\tau+ & F\left(d\left(a_{2 c}, a_{2 c+1}\right)\right) \\
& =\tau+F\left(d\left(S a_{2 c-1}, T a_{2 c}\right)\right) \\
& \leq F\left[\begin{array}{c}
\alpha\left(a_{2 c-1}, a_{2 c}\right) d\left(a_{2 c-1}, a_{2 c}\right) \\
+\beta\left(a_{2 c-1}, a_{2 c}\right)\left\{d\left(a_{2 c}, S a_{2 c-1}\right)+d\left(a_{2 c-1}, T a_{2 c}\right)\right\}
\end{array}\right] \\
& =F\left[\alpha\left(a_{2 c-1}, a_{2 c}\right) d\left(a_{2 c-1}, a_{2 c}\right)+\beta\left(a_{2 c-1}, a_{2 c}\right) d\left(a_{2 c-1}, a_{2 c+1}\right)\right] \\
& \leq F\left[\begin{array}{c}
\alpha\left(a_{2 c-1}, a_{2 c}\right) d\left(a_{2 c-1}, a_{2 c}\right) \\
+\beta\left(a_{2 c-1}, a_{2 c}\right)\left\{d\left(a_{2 c-1}, a_{2 c}\right)+d\left(a_{2 c}, a_{2 c+1}\right)\right\}
\end{array}\right]+\sigma .
\end{aligned}
$$

From (F1), we have

$$
\begin{aligned}
d\left(a_{2 c}, a_{2 c+1}\right) & <\frac{\alpha\left(a_{2 c-1}, a_{2 c}\right)+\beta\left(a_{2 c-1}, a_{2 c}\right)}{1-\beta\left(a_{2 c-1}, a_{2 c}\right)} d\left(a_{2 c-1}, a_{2 c}\right) \\
& =\lambda_{1} d\left(a_{2 c-1}, a_{2 c}\right) .
\end{aligned}
$$

On the other hand, if $a_{2 c} \leq a_{k}$

$$
\begin{aligned}
d\left(a_{2 c-1}, a_{2 c}\right) & <\frac{\alpha\left(a_{2 c-1}, a_{2 c}\right)+\beta\left(a_{2 c-1}, a_{2 c}\right)}{1-\beta\left(a_{2 c-1}, a_{2 c}\right)} d\left(a_{2 c-2}, a_{2 c-1}\right) \\
& =\lambda_{2} d\left(a_{2 c-2}, a_{2 c-1}\right) .
\end{aligned}
$$

Continuity this way, for $\lambda=\max \left\{\lambda_{1}, \lambda_{2}, \cdots, \lambda_{l}\right\}$, we deduce from inequality (37) and (38) that

$$
\begin{aligned}
& d\left(a_{2 c}, a_{2 c+1}\right)<\lambda d\left(a_{2 c-1}, a_{2 c}\right)<\cdots<\lambda^{2 c} d\left(a_{0}, a_{1}\right), \\
& d\left(a_{2 c-1}, a_{2 c}\right)<\lambda d\left(a_{2 c-2}, a_{2 c-1}\right)<\cdots<\lambda^{2 c-1} d\left(a_{0}, a_{1}\right) .
\end{aligned}
$$

From (39) and (40), we write

$$
d\left(a_{k}, a_{k+1}\right) \leq \lambda^{k} d\left(a_{0}, a_{1}\right) \quad \text { for some } \quad k \in N .
$$

Now, using (41), we have

$$
\begin{aligned}
g\left(d\left(a_{0}, a_{k+1}\right)\right) & \leq g\left(\sum_{i=1}^{k+1} d\left(a_{i-1}, a_{i}\right)\right)+\sigma \\
& =g\left(d\left(a_{0}, a_{1}\right)+\cdots+d\left(a_{k}, a_{k+1}\right)\right)+\sigma \\
& \leq g\left[\left(1+\lambda+\lambda^{2}+\cdots+\lambda^{k}\right) d\left(a_{0}, a_{1}\right)\right]+\sigma \\
& =g\left[\frac{1-\lambda^{k+1}}{1-\lambda} d\left(a_{0}, a_{1}\right)\right]+\sigma .
\end{aligned}
$$

From (b) and (c), we obtain

$$
g\left(d\left(a_{0}, a_{k+1}\right)\right) \leq g\left(\left(1-\lambda^{k+1}\right) r\right)+\sigma \leq g(\epsilon)<g(r) .
$$

Hence by (F1), we deduce that

$$
a_{k+1} \in B\left(a_{0}, r\right) .
$$

Therefore, $a_{n} \in B\left(a_{0}, r\right)$ for all $n \in N$. Now for $p \in N$,we have by (33)

$$
\begin{aligned}
\tau+F\left(d\left(a_{2 p+1}, a_{2 p+2}\right)\right)= & \tau+F\left(d\left(S a_{2 p}, T a_{2 p+1}\right)\right) \\
\leq & F\left[\alpha\left(a_{2 p}, a_{2 p+1}\right) d\left(a_{2 p}, a_{2 p+1}\right)\right. \\
& +\beta\left(a_{2 p}, a_{2 p+1}\right)\left\{d\left(a_{2 p+1}, S a_{2 p}\right)\right. \\
& \left.\left.+d\left(a_{2 p}, T a_{2 p+1}\right)\right\}\right] \\
= & F\left[\alpha\left(a_{2 p}, a_{2 p+1}\right) d\left(a_{2 p}, a_{2 p+1}\right)\right. \\
& \left.+\beta\left(a_{2 p}, a_{2 p+1}\right) d\left(a_{2 p}, a_{2 p+2}\right)\right] \\
\leq & F\left[\alpha\left(a_{2 p}, a_{2 p+1}\right) d\left(a_{2 p}, a_{2 p+1}\right)\right. \\
& \left.+\beta\left(a_{2 p}, a_{2 p+1}\right)\left\{\begin{array}{c}
d\left(a_{2 p}, a_{2 p+1}\right) \\
+d\left(a_{2 p+1}, a_{2 p+2}\right)
\end{array}\right\}\right]+\sigma .
\end{aligned}
$$

Using (a) and repeating the steps done in heorem 11, we get to the conclusion that $\left(a_{n}\right)$ is $F$-Cauchy to a point $y$ in $B\left(a_{0}, r\right)$. Proceeding in a similar way as in heorem 11 , we obtain that $y=S y=T y$

Substituting $S$ by $T$ in the previous theorem, the following result is obtained.

Corollary 17. Assume $(g, \sigma) \in \mathscr{G} \times[0, \infty), \quad(F, \tau) \in \mathscr{G} \times(0$, $\infty),(X, d)$ is an F-complete $F-M S$ and $T: X \longrightarrow X$ is a selfmap. Let $\alpha, \beta: X \times X \longrightarrow[0, \infty)$ such that $\max _{a, x \in a}\{\alpha(a, x)+2$ $\beta(a, x)\}<1$. Assume that for $a_{0} \in X$ and $r>0$, the below conditions are fulfilled:

(a) $B\left(a_{0}, r\right) \subseteq X$ is F-closed

(b) $d\left(a_{0}, a_{1}\right) \leq(1-\lambda) r$, for $a_{1} \in X$ and $\lambda=\alpha(a, x)+\beta$ $(a, x) / 1-\beta(a, x)$

(c) $\exists 0<\epsilon<r \quad$ suchas $g\left(\left(1-\lambda^{k+1}\right) r\right) \leq g(\epsilon)-\sigma$, where $k \in N$

(d) $\tau+F(d(T a, T x)) \leq F[\alpha(a, x) d(a, x)+\beta(a, x)(d(x, T$ a) $+d(a, T x))], \forall a, x \in B\left(a_{0}, r\right)$

with $\min \{d(T a, T x), d(a, x)\}>0$. Then there is a unique $y$ in $B\left(a_{0}, r\right)$ such that $T y=y$. 


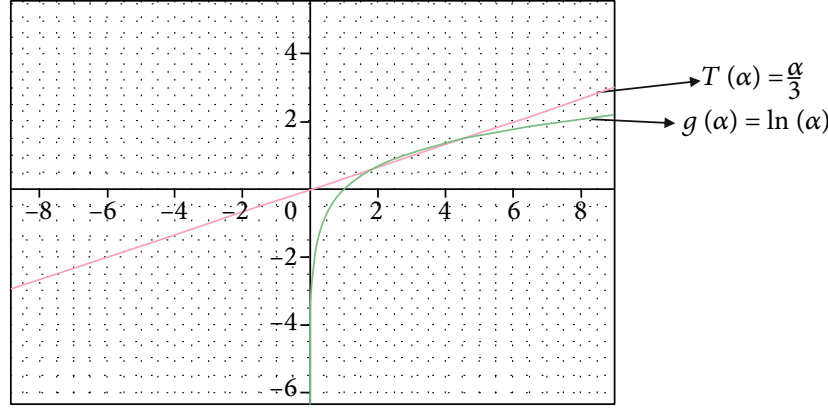

Figure 1

Example 18. Let $X=R_{0}^{+}$and $F(a)=g(a)=\ln a$. Define $T: X$ $\longrightarrow X$ by

$$
T a=\left\{\begin{array}{l}
\frac{a}{3}, \text { if } a \in[0,1] \\
a^{2}, \text { if } a \in(1, \infty) .
\end{array}\right.
$$

See Figure 1. Define $d$ by

$$
d(a, x)=\left\{\begin{array}{lll}
(a-x)^{2}, & \text { if } & (a, x) \in[0,1] \times[0,1] \\
|a-x|, & \text { if } & (a, x) \notin[0,1] \times[0,1] .
\end{array}\right.
$$

One can verify that $F$ fulfill conditions (F1) and (F2) and that $d$ is and $F$-metric.

On the other hand, define $\alpha, X \times X \longrightarrow[0, \infty)$ by

$$
\begin{aligned}
& \alpha(a, x)= \begin{cases}0, & \text { if } a=x, \\
e^{-3 / 7}, & \text { if } a \neq x,\end{cases} \\
& \beta(a, x)=\left\{\begin{array}{ll}
e^{-2}, & \text { if } x=T a \\
0, & \text { otherwise. }
\end{array} \text { and } a=T x,\right.
\end{aligned}
$$

Fix $a_{0}=r=1 / 2$, then $B\left(a_{0}, r\right)=[0,1]$. Clearly, $B\left(a_{0}, r\right)$ is $F$-closed hence is satisfied. Now, since $a_{0} \neq a_{1}$, therefore, $\alpha(a, x)=e^{-3 / 7}$ and $\beta(a, x)=0$, which implies that $\lambda=\alpha(a, x)$ and

$$
d\left(a_{0}, a_{1}\right)=d\left(a_{0}, T a_{0}\right)=\left(\frac{1}{2}-\frac{1}{6}\right)^{2}<\left(1-e^{-3 / 7}\right) \frac{1}{2}=(1-\lambda) r
$$

Therefore, condition (b) is obeyed. Moreover, assume $k=1$, then $g\left(\left(1-\lambda^{k+1}\right) r\right)=\ln \left(\left(1-\left(e^{-3 / 7}\right)^{2}\right)(1 / 2)\right)=\ln (2 / 5)-\ln$ $(2 / 7)=g(\varepsilon)-\sigma$ is satisfied. i.e., $\varepsilon=(2 / 5) \leq(1 / 2)=r$ and $\sigma$ $=\ln (2 / 7)$. In a similar way, for each $k \in N, \exists$ some $0<\epsilon<$ $r$ and $\sigma$ satisfying condition (c). Now checking for condition (d), we have two cases:

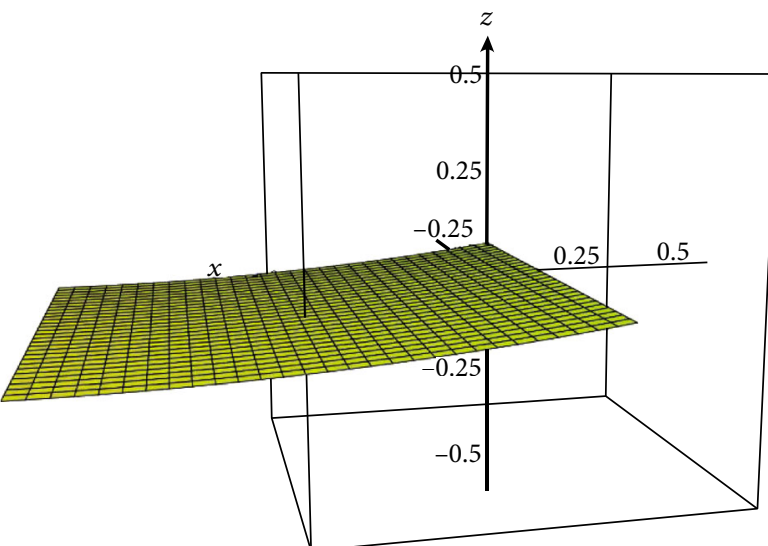

Figure 2

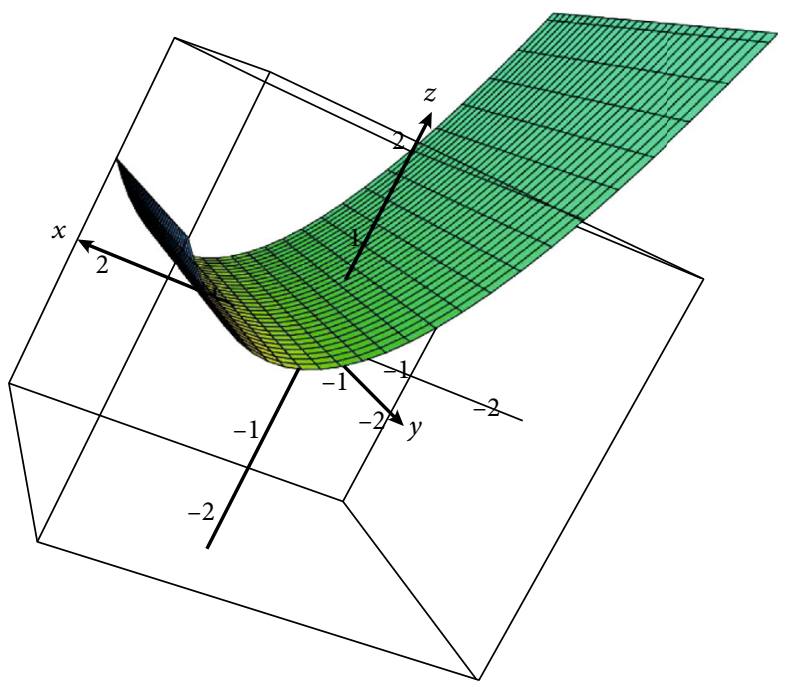

Figure 3

Case 19. If $(a, x) \in B\left(a_{0}, r\right) \times B\left(a_{0}, r\right)$, then

$$
\begin{aligned}
F(d(T a, T x)) & =\ln \left(\left(\frac{a}{3}-\frac{x}{3}\right)^{2}\right)<\ln \left(e^{-3 / 7}(a-x)^{2}\right) \\
& =F[\alpha(a, x) d(a, x)+\beta(a, x)\{d(a, T a)+d(x, T x)\}] .
\end{aligned}
$$

Figures 2-4 illustrate this inequality, where

$$
\begin{aligned}
\tau \in & \left(\frac{2}{7}, \ln \left(e^{-3 / 7}(a-x)^{2}\right)-\ln \left(\left(\frac{a}{3}-\frac{x}{3}\right)^{2}\right)\right) \\
& =\left(\frac{2}{7}, \ln \frac{e^{-3 / 7}}{1 / 9}\right)=\left(\frac{2}{7}, \ln \left(9 e^{-3 / 7^{3}}\right) .\right.
\end{aligned}
$$

Therefore, for all $(a, x) \in B\left(a_{0}, r\right) \times B\left(a_{0}, r\right)$, condition (d) is also satisfied. 


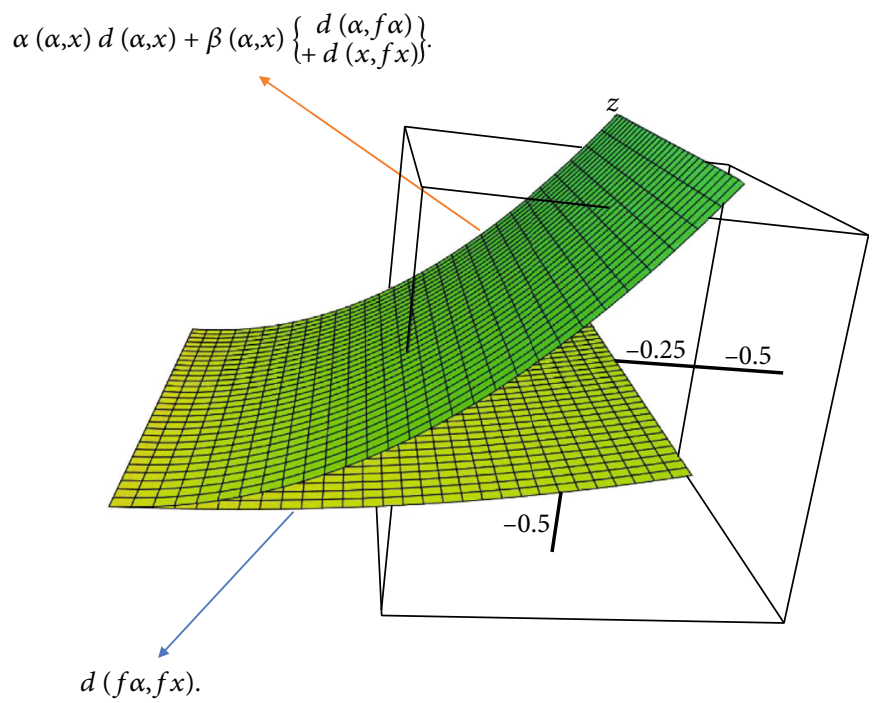

Figure 4

Case 20. If $(a, x) \notin B\left(a_{0}, r\right) \times B\left(a_{0}, r\right)$, e.g., $a=2$ and $x=3$, then

$$
\begin{aligned}
F(d(T a, T x)) & =\ln \left(\left|2^{2}-3^{2}\right|\right)>\ln \left(e^{-3 / 7}|(2-3)|\right) \\
& =F(\alpha(a, x) d(a, x)) \\
& =F\left[\begin{array}{c}
\alpha d(a, x) \\
+\beta d(a, T a)+\gamma d(x, T x)
\end{array}\right] \cdot F(d(T a, T x)) \\
& =\ln \left(\left|2^{2}-3^{2}\right|\right)>\operatorname{In}\left(e^{-3 / 7}|2-3|\right) \\
& =F(\alpha(a, x) d(a, x)) \\
& =F\left[\begin{array}{c}
\alpha d(a, x) \\
+\beta d(a, T a)+\gamma d(x, T x)
\end{array}\right] .
\end{aligned}
$$

Hence, condition (b) holds only for $B\left(a_{0}, r\right)$ and not on $X \times X$. Moreover, $0 \in B\left(a_{0}, r\right)$ is the fixed point of $T$. Given below Figure 1 shows two maps $f(a)=a / 3$ and $g(a)=\ln (a)$

Figures 2 and 3 are 3D graphs of the functions $z=d$ $(f a, f x)=((a / 3)-(x / 3))^{2} \quad$ and $\quad z=d(a, x)=(a-x)^{2}$, respectively.

Multiplying $\alpha(a, x)=e^{-3 / 7}$ to $d(a, x)$ of the contractive inequality and combining Figures 2 and 3 , we get the below 3D graph which clearly demonstrate that the graph of $\alpha(a, x) d(a, x)$ is dominating the graph of $d(f a, f x)$.

$$
\begin{gathered}
\alpha(a, x) d(a, x)+\beta(a, x)\left\{\begin{array}{c}
d(a, f a) \\
+d(x, f x)
\end{array}\right\} . \\
d(f a, f x) .
\end{gathered}
$$

As we see in Figure $1, \ln x$ is an increasing function. Therefore, it will not change the inequality, i.e., the right side of the inequality will still be dominant. Note that $z$ -axis represents the values of the function $g$, and it can be observed that for every value of $a$ and $x, F(d(f a, f x))$ $<F(\alpha(a, x) d(a, x))$ and hence satisfy the inequality of the above example.

Corollary 21. Assume $(g, \sigma) \in \mathscr{G} \times[0, \infty),(F, \tau) \in \mathscr{G} \times(0, \infty)$ such that $\sigma<\tau$ and $(X, d)$ is an F-complete F-MS. Let $S$. $T: X \longrightarrow X$ are self-maps and $k: X \times X \longrightarrow[0,1)$. Assume that for $a_{0} \in X$ and $r>0$, the below conditions are fulfilled:

(a) $B\left(a_{0}, r\right) \subseteq X$ is F-closed

(b) $\tau+F(d(S a, T x)) \leq F[k(a, x) / 2(d(a, S a)+d(x, T x))]$, for all $a, x \in B\left(a_{0}, r\right)$

(c) $d\left(a_{0}, a_{1}\right) \leq(1-\lambda) r$, for $a_{1} \in X$ and $\lambda=k(a, x) / 2-k$ $(a, x)$

(d) $\exists 0<\epsilon<r$ such as $g\left(\left(1-\lambda^{n+1}\right) r\right) \leq g(\epsilon)-\sigma$, where $n \in N$

Then $S y=T y=y$ for a unique $y$ in $B\left(a_{0}, r\right)$.

\section{Application to Functional Equations}

This section discusses the application of our results in finding a common solution of functional equations that are used in dynamic programming.

The study of dynamic programming splits into two parts. A state space is a set of parameters of various states, i.e., initial states, transitional states, and action states. On the other hand, a decision space is a series of actions taking place for finding the possible solution to the indicated problem. The problem of dynamic program is transformed into functional equations:

$$
g(x)=\max _{y \in X}\{H(x, y)+J(x, y, g(\eta(x, y)))\} \quad \text { for } \quad x \in A,
$$




$$
f(x)=\max _{y \in X}\{H(x, y)+K(x, y, g(\eta(x, y)))\} \quad \text { for } \quad x \in A \text {, }
$$

where $U$ and $V$ are Banach spaces such that $A \subseteq U$ and $X \subseteq V$ and

$$
\begin{gathered}
\eta: A \times X \longrightarrow A, \\
H: A \times X \longrightarrow R, \\
J, K: A \times X \times R \longrightarrow R .
\end{gathered}
$$

Assume $A$ and $X$ are state space and decision spaces, respectively. Assume $W(A)$ denotes a set of all-bounded realvalued maps on $A$. Let $h \in W(A)$ and say ||$h \|=\max _{x \in A}|h(x)|$. Then, $(W(A),\|\cdot\|)$ is a Banach space and $d$ is the metric defined as

$$
d(h, k)=\max _{x \in A}|h(x)-k(x)|
$$

Suppose the following conditions are satisfied:

$\left(C_{1}\right): H, J$, and $K$ are bounded.

$\left(C_{2}\right)$ : For $x \in A$ and $h \in W(A)$, define $P, Q: W(A) \longrightarrow$ $W(A)$ by

$$
\begin{aligned}
& P h(x)=\max _{y \in X}\{H(x, y)+J(x, y, h(\eta(x, y)))\} \text { for } x \in A, \\
& Q h(x)=\max _{y \in X}\{H(x, y)+K(x, y, h(\eta(x, y)))\} \text { for } x \in A .
\end{aligned}
$$

Observe that the functions $H, J$, and $K$ are bounded hence $P$ and $Q$ are well-defined.

$\left(C_{3}\right):$ For $\sigma<\tau: R_{+} \longrightarrow R_{+},(x, y) \in A \times X, h, k \in W(A)$ and $t \in A$, we have

$$
|J(x, y, h(t))-K(x, y, k(t))| \leq e^{-\tau} M(h, k),
$$

where

$$
M(h, k)=\alpha d(h, k)+\beta d(k, P h)+\gamma d(h, Q k)
$$

for $\alpha, \beta, \gamma \in[0, \infty)$ such that $\alpha+\beta+\gamma<1$, where min $\{d(P h$, $Q k), d(h, k)\}>0$.

Based on the above hypothesis, we present the below theorem.

Theorem 22. Let $\left(C_{1}\right)-\left(C_{3}\right)$ are satisfied, then at most one bounded common solution exists for Equations (54) and (55).

Proof. We know by Lemma 10 that $(W(A), d)$ is an F-complete $F$-MS, $d$ is stated by $(57)$ and $\left(C_{1}\right)$ say that $P$ and $Q$ are self-maps on $W(A)$. Choose any positive number $\omega$ and $h_{1}, h_{2} \in W(A)$. Take $x \in A$ and $y_{1}, y_{2} \in X$ such that

$$
\begin{gathered}
P h_{c}<H\left(x, y_{c}\right)+J\left(x, y_{c}, h_{c}\left(\eta\left(x, y_{c}\right)\right)\right)+\omega, \\
Q h_{c}<H\left(x, y_{c}\right)+K\left(x, y_{c}, h_{c}\left(\eta\left(x, y_{c}\right)\right)\right)+\omega,
\end{gathered}
$$

$$
\begin{gathered}
P h_{1} \geq H\left(x, y_{2}\right)+J\left(x, y_{2}, h_{1}\left(\eta\left(x, y_{2}\right)\right)\right), \\
Q h_{2} \geq H\left(x, y_{1}\right)+K\left(x, y_{1}, h_{2}\left(\eta\left(x, y_{1}\right)\right)\right) .
\end{gathered}
$$

Then using (61) and (64), we get

$$
\begin{aligned}
& P h_{1}(x)-Q h_{2}(x) \\
& \quad<J\left(x, y_{1}, h_{1}\left(\eta\left(x, y_{1}\right)\right)\right)-J\left(x, y_{1}, h_{2}\left(\eta\left(x, y_{1}\right)\right)\right)+\omega \\
& \quad \leq\left|K\left(x, y_{1}, h_{1}\left(\eta\left(x, y_{1}\right)\right)\right)-K\left(x, y_{1}, h_{2}\left(\eta\left(x, y_{1}\right)\right)\right)\right|+\omega \\
& \quad \leq e^{-\tau} M\left(h_{1}(x), h_{2}(x)\right)+\omega .
\end{aligned}
$$

Similarly, by (62) and (63), we get

$$
Q h_{2}(x)-P h_{1}(x)<e^{-\tau} M\left(h_{1}(x), h_{2}(x)\right)+\omega \text {. }
$$

Combining the above two inequalities, we get

$$
\left|P h_{1}(x)-Q h_{2}(x)\right|<e^{-\tau} M\left(h_{1}(x), h_{2}(x)\right)+\omega
$$

for all $\omega>0$. Hence,

$$
d\left(P h_{1}(x), Q h_{2}(x)\right) \leq e^{-\tau} M\left(h_{1}(x), h_{2}(x)\right)
$$

that is,

$$
d\left(P h_{1}, Q h_{2}\right) \leq e^{-\tau} M\left(h_{1}, h_{2}\right)
$$

for each $x \in A$. Applying logarithms on both sides, we get

$$
\ln \left(d\left(P h_{1}, Q h_{2}\right)\right) \leq \ln \left(e^{-\tau} M\left(h_{1}, h_{2}\right)\right)
$$

This shows that $F: R_{+} \longrightarrow R$ defined as $F(x)=\ln x$ is a member of $\mathscr{G}$, and

$$
\tau+F\left(d\left(P h_{1}, Q h_{2}\right)\right) \leq F\left(M\left(h_{1}, h_{2}\right)\right) .
$$

As every condition in Theorem 11 is fulfilled, therefore using heorem $11 \mathrm{P}$ and $\mathrm{T}$ have a unique common and bounded solution of the Equations (54) and (55).

\section{Conclusion}

This article instigated the establishment of fixed point result of Reich type $F$-contractions, while imposing the contractive conditions on both the whole $F$-MS as well as only on a subset ( $F$-closed ball) of the F-MS. However, the constants in the inequality of Reich type contractive conditions are replaced by real-valued functions. The validity of the inequality is verified graphically, making the results clearer and more certain. At last, the use of our results in assuring the existence of a solution to the functional equation is described.

\section{Data Availability}

No data were used to support the study. 


\section{Conflicts of Interest}

The authors declare that they have no competing interests

\section{Authors' Contributions}

All the authors contributed equally to the research.

\section{References}

[1] M. R. Alfuraidan and M. A. Khamsi, "On multivalued Gmonotone Ciric and Reich contraction mappings," Filomat, vol. 31, no. 11, pp. 3285-3290, 2017.

[2] N. Hussain, M. Arshad, M. Abbas, and N. Hussain, "Generalized dynamic process for generalized (f,L)-almost Fcontraction with applications," Journal of Nonlinear Sciences and Applications, vol. 9, pp. 1702-1715, 2016.

[3] M. Arshad, E. Ameer, and A. Hussain, "Hardy-Rogers-type fixed point theorems for $\alpha$-GF-contractions," Archivum Mathematicum, vol. 51, pp. 129-141, 2015.

[4] M. Cosentino and P. Vetro, "Fixed point results for F-contractive mappings of Hardy-Rogers type," Filomat, vol. 28, no. 4, pp. 715-722, 2014.

[5] G. Minak, A. Halvaci, and I. Altun, "Ćirić type generalized Fcontractions on complete metric spaces and fixed point results," Filomat, vol. 28, no. 6, pp. 1143-1151, 2014.

[6] T. Rasham, A. Shoaib, B. Alamri, and A. Asif, "Fixed point results for $\alpha *-\psi$-dominated multivalued contractive mappings endowed with graphic structure," Mathematics, vol. 7, p. 307, 2019.

[7] S. B. Nadler Jr., "Multivalued contraction mappings," Pacific Journal of Mathematics, vol. 30, pp. 475-488, 1969.

[8] S. Reich, "Some remarks concerning contraction mappings," Canadian Mathematical Bulletin, vol. 14, no. 1, pp. 121-124, 1971.

[9] D. Wardowski, "Fixed points of a new type of contractive mappings in complete metric spaces," Fixed Point Theory and Applications, vol. 2012, no. 1, Article ID 94, 2012.

[10] D. Klim and D. Wardowski, "Fixed points of dynamic processes of set-valued F-contractions and application to functional equations," Fixed Point Theory and Applications, vol. 2015, no. 1, Article ID 22, 2015.

[11] M. Nazam, C. Park, A. Hussain, M. Arshad, and J. R. Lee, "Fixed point theorems for F-contractions on closed ball in partial metric spaces," Journal of Computational Analysis and Applications, vol. 27, pp. 759-769, 2019.

[12] M. Sgroi and C. Vetro, "Multi-valued F-contractions and the solution of certain functional and integral equations," Filomat, vol. 27, no. 7, pp. 1259-1268, 2013.

[13] A. Shoaib and A. Asif, "Generalized dynamic process for generalized multivalued F-contraction of Hardy Rogers type in bmetric spaces," Turkish Journal of Anal. and Number Theory, vol. 6, pp. 43-48, 2018

[14] A. Asif, M. Nazam, M. Arshad, and S. O. Kim, "F-metric, Fcontraction and common Fixed-Point theorems with applications," Mathematics, vol. 7, no. 7, p. 586, 2019.

[15] D. Wardowski and N. V. Dung, "Fixed points of F-weak contractions on complete metric spaces," Demonstratio Mathematics, vol. 47, no. 1, pp. 146-155, 2014.
[16] D. Wardowski and N. V. Dung, "A note on fixed point theorems in metric spaces," Carpathian Journal of Mathematics, vol. 31, no. 1, pp. 127-134.

[17] M. Jleli and B. Samet, "On a new generalization of metric spaces," Journal of Fixed Point Theory and Applications, vol. 20, no. 3, p. 128, 2018.

[18] A. Hussain and T. Kanwal, "Existence and uniqueness for a neutral differential problem with unbounded delay via fixed point results," Transactions of A. Razmadze Mathematical Institute, vol. 172, no. 3, pp. 481-490, 2018. 